\title{
COMPARATIVE STUDY OF PLANNING AND SCHEDULING OF A CONSTRUCTION PROJECT USING MICROSOFT PROJECT
}

\author{
Shruti Singh ${ }^{1}$, Shweta Istape $^{2}$, Amruta Surve $^{3}$, Sahil Pandey $^{4}$, Avinash Singh $^{5}$, Sangram More $^{6}$ \\ ${ }^{1}$ Assistant Professor, MGMCET, Kamothe, University of Mumbai, Navi Mumbai, India \\ ${ }^{2}$ Assistant Professor, MGMCET, Kamothe, University of Mumbai, Navi Mumbai, India \\ ${ }^{3}$ Student, MGMCET, Kamothe, University of Mumbai, Navi Mumbai, India \\ ${ }^{4}$ Student, MGMCET, Kamothe, University of Mumbai, Navi Mumbai, India \\ ${ }^{5}$ Studentr, MGMCET, Kamothe, University of Mumbai, Navi Mumbai, India \\ ${ }^{6}$ Student, MGMCET, Kamothe, University of Mumbai, Navi Mumbai, India
}

\begin{abstract}
Project Management is the application of knowledge, tools, techniques and skills to any project activities to meet the requirements of the project. Usually many construction industries in India do not practice the use of project management properly. Poorly managed projects usually result in a huge amount to all stakeholders not just financially but also psychologically and emotionally as well as it also consumes a lot of time. This has greatly motivated us to carry out the working and functioning of a project using modern project management tool, one of which is MSP (Microsoft Project). This paper consists of planning and scheduling of various activities involved in a construction project using MSP. The presented work clearly gives the difference between carrying out the work using MSP and traditional planning techniques. The presented work has been carried out on a residential building in Mumbai and it clearly shows how the use of MSP software can speed up the construction making it cost effective. Methodology for the presented work includes defining the problem statement, collection of data and finally coming to the conclusion.
\end{abstract}

Keywords: - Project Management, Scheduling, Planning, Activities, Microsoft Project. $* * *$

\section{INTRODUCTION}

Project management is the planning, monitoring and scheduling of all aspects of a project and the motivation of all those involved in it to achieve the project objectives on time and to the specified cost, quality and performance. Construction project management is the art and science of managing all aspects of the project to achieve the project mission objectives, the specific time, budget cost and predefined quality specifications; working efficiently and effectively in the changing project environment with due regards to construction worker's safety and health. Project management is essentially aimed at producing an end product that will effect some change for the benefit of the organisation that instigated the project. It is the initiation, planning and control of a range of tasks required to deliver this end product.

\subsection{Importance of Construction Project Planning}

a) Planning helps to minimize the cost by optimum utilization of available resources.

b) Planning reduces irrational approaches, duplication of works and inter departmental conflicts.

c) Planning encourages innovation and creativity among the construction managers.

d) Planning imparts competitive strength to the enterprise.

\subsection{Purpose of Scheduling}

a) A Schedule is the reflection of the plan.

b) It is the determination of time and sequence of operation in the project and their assembly to give the overall completion time.

c) The process of scheduling uncovers flaws in the plan, leading to easy revision of the plan.

\subsection{Microsoft Project}

Microsoft Project is a project management software product, developed and sold by Microsoft. It is designed to assist a project manager in developing a plan, assigning resources to tasks, tracking progress, managing the budget, and analyzing workloads. MS project can be used as a standalone tool for tracking project progress or it can be used for tracking complex project distributed in many geographical areas and managed by a number of project managers. Microsoft project is designed to assist a project manager in;

1. Developing a plan,

2. Assigning resources to tasks,

3. Tracking progress,

4. Managing budget and

5. Analysing workloads. 


\section{BRIEF OVERVIEW OF LITERATURE}

Rhuta Joshi and Prof. V.Z. Patil analysed the project management technique by scheduling various construction activities, allocation of resources and resource leveling using Microsoft Project 2013 for residential building. The study was carried out in two phases. In first phase data was collected from site and quantities were calculated as per drawing and required manpower was calculated. In second phase of construction activities was defined in MSP 2013. The result was as resource decreases duration increased by $10.38 \%$ and cost by $0.94 \%$.

Nikhil R. Mahajan and M. V. Bhogone (2017) The methodology adapted by them was to compare Microsoft Project and Traditional Method. Schedule was prepared for both conventional and prefabrication method. A residential building was taken for comparison. The software used was MSP, the duration required for completion of project was collected from respective company. The comparison was made by comparing the total time required for completion by using critical path method with MSP project. The result shows that the total duration for both the conventional and prefabrication method and prefab construction for individual house and double story the required cost is $13 \%$ more than conventional but it reduces the project duration by 63 days.

Wallance Agyei The study was aimed at finding the difference between the cost and minimum expected time that will be required to complete the project. Both CPM and PERT techniques were used for analysis and from the result it was concluded that schedule proposed by bus provides much shorter completion time as compared to the actual time taken by the process.

Rashmi J.V. and Amey A. Kelkar (2017) They analysed the planning and scheduling of multi-storeyed building in two phase by conventional execution approach \& again analysis of same building was carried out by applying MSP to compare the result for justification. For their study they considered $\mathrm{G}+3$ with basement and the type of RCC frame structure, to estimate the overall cost and time required to execute a multistoreyed residential building. The result of their study showed that proper manage of project management skills and technique reduces the time by $23.2 \%$ and cost by $3.14 \%$.

E. Suresh kumar and S. Krishnamoorthi (2015) In their study they focused on the scheduling using MSP and earns value analysis for an apartment building. Thereby time required for the process of cost overrun is avoided. Project schedule is considered as core of the project plan, and the purpose of the project schedule is to show the organization how the work will be performed to uncover the mistakes. After completion of project it has been observed that there is more difference between budget cost and actual cost, cost increases as the material price increases. Earned value analysis is carried out in order to find the variance cost of the project.

\section{METHODOLOGY USED FOR THE RESEARCH}

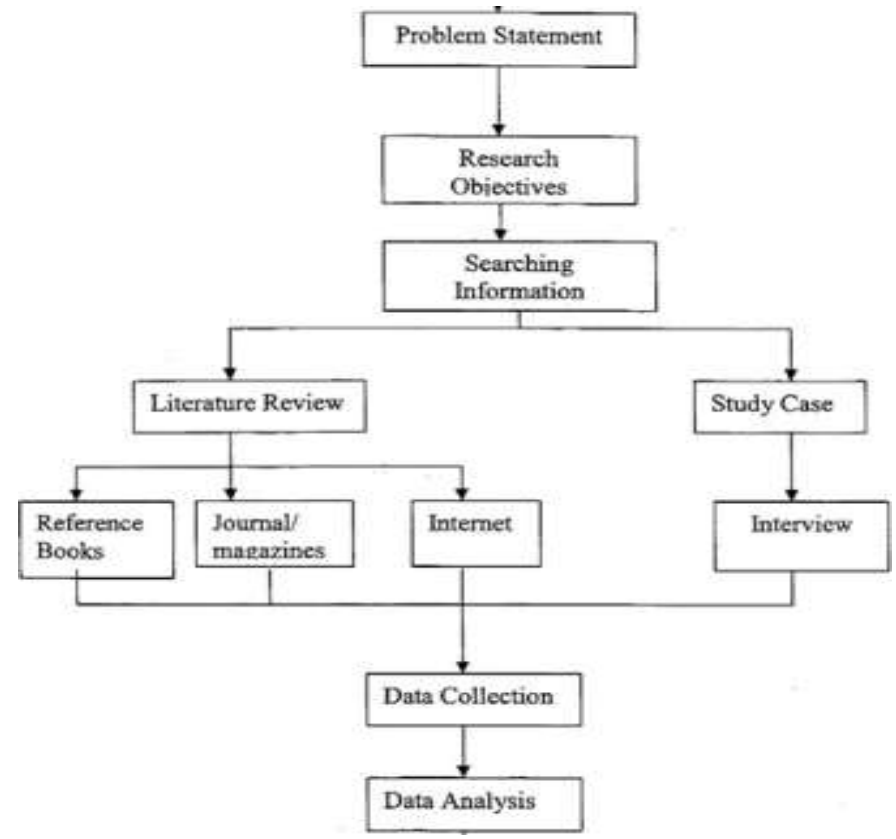

Fig-1. Research Methodology Flowchart.

\subsection{Problem Statement}

Many of the construction industries, nowadays, are facing problems with respect to the time consumed, cost incurred and delays occurring in completing a construction project. Usually these problems occur when a project has not been planned properly. Project planning involves and explains the customers about the detailing strategy that should be followed for the project completion. The primary and important uses of planning the project are to facilitate communication among stakeholders, to plan the documents related to planning assumptions and decisions, and also to develop document approved scope, cost and schedule baselines.

Construction project management is the art and science of managing all aspects of the project to achieve the project mission objectives, the specific time, budget cost and predefined quality specifications; working efficiently and effectively in the changing project environment with due regards to construction worker's safety and health. Mission of creating a construction facility or services, with predetermined performance objectives define in terms of quality specifications, completion time, budget cost and other specified constraints.

The presented work reviewed concepts of activities sequence that are used for construction of buildings, techniques of scheduling used in the Microsoft project planner and the development of construction planning and scheduling of building construction. This project study gives the information about the project from the starting to the end of the project. After the completion of the study, we can able to know that what we have to do on project site before starting the project, in the middle of the project and after completion of the project of construction. 


\subsection{Research Objective}

The objective of the research work is to plan and develop construction facilities in such a way that proper systematic planning of construction activities can be achieved. It was also aimed at providing good and proper quality and quantity of machine, men, materials etc., at right stage of the project. And lastly to inform the management, ahead of time, about the difficulties or the various awkward position expected to crop up later in the project.

The following objectives were set for the project: -

1. To identify the sequence of construction activities followed for the construction of the residential building.

2. To determine the technique of planning and scheduling that has been used by the construction company for the completion of the construction work.

3. To develop a module of scheduling using Microsoft Project Software.

4. To identify proper execution of Planning and Scheduling.

5. To know the steps required before and after completion of the project.

6. To try the project and analyse the reason for delays and increase in estimated budget etc.

7. To investigate defects in the planning and scheduling procedure of the organisation and suggest suitable improvements in their methods.

\subsection{Searching Information}

\subsubsection{Literature Review}

Various literatures were reviewed for the projects explained earlier.

\subsubsection{Reference Books}

Following books were referred for the presented work: 'Project Management: A systems Approach to Planning, Scheduling and Controlling', 'A Guide to the Project Management Body of Knowledge', 'Brilliant Project Management: What the Best Project Managers know, do and say'.

\subsubsection{Journals/Magazines}

Some of the International Journals such as Science Direct, Indian Journal of research, IJRET, IOSR for Mechanical and Civil engineering, were also referred to know about the work done related to Microsoft Project software in the past.

\subsection{Case Study}

The case study for the project involves the planning and scheduling of a residential building. For this case G+6 residential building, located at Gundage, Karjat, Maharashtra, India was selected. The site details, plans and drawings etc were utilized to achieve the goals of the project. Type of Structure -Reinforced Cement Concrete Structure (RCC) G +6 Residential Building.

\subsubsection{Interview}

For the presented work we had scheduled an interview with the Senior Engineer and Project coordinator of the respective construction company in Mumbai, India. The main motive of the interview was to determine the problems that took place during the execution of the project. The problems can be anything related to labour fatigue, duration, delay in project completion, resource under allocation or over allocation, etc. The interview also helped in knowing the sequence of activities planned and followed while execution.

\subsection{Data Collection}

Data collection for any project is very important for corelating the different data and then using in working with Microsoft Project 2010. This data collection can include the starting date of different activities, their finish dates and their delays, relationship between tasks and activities, amount of resources used for different activites.

\subsection{Data Analysis}

The data collected from the company was analysed and divided into activities and sub activities. The activities were analysed using Work Breakdown Structure and proper planning was done using Microsoft Project.

\section{WORKING WITH MICROSOFT PROJECT AND}

\section{RESULT}

\subsection{Construction Site Details}

Table-1: Excavation Quantity.

\begin{tabular}{|l|l|l|l|l|l|}
\hline $\begin{array}{l}\text { Sr. } \\
\text { No. }\end{array}$ & Length & Breadth & Height & Quantity & Remarks \\
\hline 1 & 20 & 12 & 3 & 720 & CU.M \\
\hline
\end{tabular}

1. Material used footing and column below plinth.

2. Grade of concrete $=$ M25(1:1:2)

3. Cement bags for footing $=450$

4. Cost of cement per bag = Rs.330

5. Cost of steel per kg $=$ Rs. 40

6. Cement used on site $=\quad$ Birla A1 cement (Rs 330 Per bag)

7. Excavation and layout was done in 10 days

8. Dr.fixit WP was used for water proofing of the foundation and lw was used for strength.

9. An estimated 35 days was completely utilized for foundation work

Table -2: Manpower associated with footing

\begin{tabular}{|l|l|l|l|}
\hline $\begin{array}{l}\text { Sr. } \\
\text { No }\end{array}$ & Machinery suppliers & No. & $\begin{array}{l}\text { Overall cost } \\
\text { (in Rs) }\end{array}$ \\
\hline 1. & JCB & 2 & $750 / \mathrm{HR}$ \\
\hline 2. & Poklan & 1 & $1100 / \mathrm{HR}$ \\
\hline 3. & Breaker poklan & 1 & $1300 / \mathrm{HR}$ \\
\hline
\end{tabular}


Table-3: Steel reinforcement details

\begin{tabular}{|l|l|l|l|}
\hline Member & $\begin{array}{l}\text { Diameter Of Steel } \\
\text { Reinforcement } \\
\text { Bar }\end{array}$ & $\begin{array}{l}\text { Concrete } \\
\text { Quantity } \\
\text { CU-M }\end{array}$ & $\begin{array}{l}\text { Steel } \\
\text { Quantity } \\
\text { Ton }\end{array}$ \\
\hline Column & $\begin{array}{l}8 \mathrm{~mm}, 12 \quad \mathrm{~mm}, \\
16 \mathrm{~mm}\end{array}$ & 144 & 18.4 \\
\hline Beam & $8 \mathrm{~mm}, 16 \mathrm{~mm}, 20 \mathrm{~mm}$ & 211 & 22.7 \\
\hline Slab & $10 \mathrm{~mm}$ & 110.27 & 49.5 \\
\hline
\end{tabular}

Table-4: Classification of Manpower

\begin{tabular}{|l|l|l|}
\hline Type of workers & $\begin{array}{l}\text { No. of labours } \\
\text { per slab }\end{array}$ & Cost in Rupees \\
\hline Unskilled labour & 8 & 350 \\
\hline Skilled labour & 4 & 500 \\
\hline Carpenter & 4 & 550 \\
\hline Mason & 6 & 1000 per day \\
\hline Fitter & 2 & 550 \\
\hline
\end{tabular}

Table-5: Material Used

\begin{tabular}{|l|l|l|l|}
\hline Materials & $\begin{array}{l}\text { Cost (in } \\
\text { Rs) }\end{array}$ & $\begin{array}{l}\text { Quantity } \\
\text { used per slab }\end{array}$ & $\begin{array}{l}\text { Cost per } \\
\text { slab(in Rs) }\end{array}$ \\
\hline $\begin{array}{l}\text { Shuttering } \\
\text { plates }\end{array}$ & $\begin{array}{l}50 \text { per } \\
\text { plate }\end{array}$ & $\begin{array}{l}730 \text { plates } \\
7000 \text { per } \\
\text { brass }\end{array}$ & 16500 \\
\hline Sand & $\begin{array}{l}2500 \text { prass } \\
\text { brass }\end{array}$ & 49000 \\
\hline Aggregate & $\begin{array}{l}330 \text { per } \\
\text { bag }\end{array}$ & 650 bags & 214500 \\
\hline Cement bag & $\begin{array}{l}600 \text { per } \\
\text { tanker }\end{array}$ & 3 tankers & 1800 \\
\hline Water tanker & $\begin{array}{l}\text { to000 per } \\
\text { ton tons }\end{array}$ & 120000 \\
\hline Steel &
\end{tabular}

\subsection{Scheduling with Gantt Charts and WBS}

Gantt charts are a special type of view that are used extensively in project management. In MSP, the left side of the gantt chart contains a sheet view, and the right side contains a bar graph along a timescale. In msp, the gantt chart is developed depending upon the duration we enter for respective task and how to efficiently establish task dependencies.

As we refine the durations, link tasks, and possibly enter date constraints or assign recourses, Project 2010 calculate schedule to reflect those controls automatically scheduled task are marked with the gantt bar icon in the task.

\begin{tabular}{|c|c|c|c|c|c|c|c|c|c|}
\hline & \multirow[t]{2}{*}{ Tese Kane } & \multirow{2}{*}{$\begin{array}{l}\text { Actal } \\
\text { Aarnim }\end{array}$} & \multirow[t]{2}{*}{ Stat } & \multirow[t]{2}{*}{ Finit } & \multirow[t]{2}{*}{ Natial Cond } & \multirow[t]{2}{*}{ Preder. } & \multirow[t]{2}{*}{ Resourct vartio } & ant.2517 & \multirow{2}{*}{$\frac{\operatorname{ann} 3,}{4}$} \\
\hline & & & & & & & & Int $\mathrm{Mar} \mathrm{Var}$ & \\
\hline 0 & - FNAL YEAR BE NS PAOJECT & 186.87 days & Fri 03-03-17 & Fit $08-12-17$ & 3. $3,647,430.00$ & & & & \\
\hline 1 & BE PROACT & $166.87 \mathrm{dry}$ & Files-03-1) & Fin $08-12-1$ & रु.3,547,693,00 & & & & \\
\hline 2 & MLESTONE & Odge & Finos-03-17 & Fii03-08-17 & $\delta 0.00$ & & 7 & of $63-03$ & \\
\hline 3 & PADECT START & Odren & Hios-03:1? & Fri03-03-1) & 70,00 & & & +0303 & \\
\hline 4 & - Evanegaing & 5 days & EA 03-03-17 & The 09.03 .17 & रु52.750.00 & & & 4 & \\
\hline 5 & PRRCUREMENT OF DQAWNOS & Int & Fifos-03-17 & Tha 09-03-17 & 552,750000 & 2,3 & ADCHITECTUSE, DESGN ENGNEER,dLaftman & $0303 ; 00.03$ & \\
\hline 6 & Psocuasvent OF MatenaLS & 2 days & Fri $10-03-17$ & Mon 25-03-17 & $\delta 2000.00$ & & & $\phi$ & \\
\hline 7 & NATERIAL ORDER AND OELKERY. & $2 \mathrm{dqp}$ & fri $10-03-17$ & Mon $13-03-17$ & $\gamma_{2,00000}$ & 5 & SUPEMISCR,UNSDLLE LACLA[4] & $10037,13-03$ & \\
\hline e & - CONSTRuction & 17989 day & Tue 14-03.27 & Fincos.12-17 & है $3,592,603000$ & & & & \\
\hline g: & PGEPARATION OF SITE & 15 den & Tue $14 \cdot 03 \cdot 17$ & Man $0304 \cdot 17$ & रु 45,250000 & & & $\rightleftharpoons$ & \\
\hline$w$ & MASENG BCUNDGates & $1 d q$ & Tue $1403-17$ & Tue $14-03-17$ & 83,200003 & 7 & 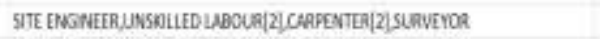 & $1403(1403$ & \\
\hline n & SURVEYWG & $2 \mathrm{dqp}$ & Wed $15-03-17$ & The $1601-17$ & $\varnothing 15,400,00$ & $\$ 0,5$ & 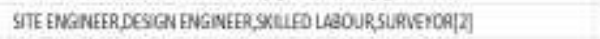 & $15-63\rceil 16-03$ & \\
\hline$n$ & Sitf OfFict & Idy & Wed $15 \cdot-19 \cdot 17$ & Wed $1503: 17$ & $\gamma_{1,2009}$ & 10 & 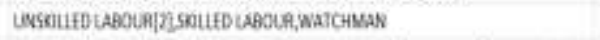 & $15-63$ is $15-3$ & \\
\hline 23 & WATER AAPANGEMENTS & $0.5 \mathrm{dap}$ & Fil $13-03-17$ & Fil $12-03-17$ & $\bar{t}_{1,40000}$ & II & 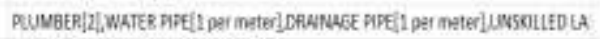 & $1708: 17.03$ & \\
\hline 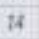 & EIECTRCITY MaRWNGANENTS & Hdan & Thu $16-03-17$ & The $78-03-17$ & $\$ 15,40000$ & 12 & BECTNGTWNUNSKILEDLAEOUR|2| & $16-03 \quad 030.03$ & \\
\hline is & STEE YaCos wO GCCOWN & $1 \mathrm{~d} y$ & Mos 2003-17 & Mon 20-03-17 & 8,20000 & 13 & 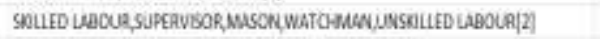 & $20,001,2003$ & \\
\hline If & MANGATE ANDFENCWS & $2 \mathrm{dan}$ & fir31-03:17 & Mon $63-417$ & 86,40000 & 34 & 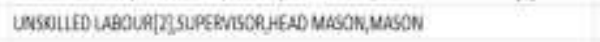 & $31-63703.04$ & \\
\hline$n$ & -sussmucture & 62.5 dap & Tue 21:03-17 & Thu $15.06 \cdot 17$ & रु 10:4, 450.00 & & & & \\
\hline Is & UNE OUT AND EXCAVATION & 15 tepn & Tue 21.03.17 & Mon: $: 0.0417$ & रु $147,000.90$ & & & $\Rightarrow$ & \\
\hline 79 & BULCONG LwE OUT & 2 deps & $T u=21-02-17$ & Wed $2203-17$ & $\delta 4,00000$ & 15 & 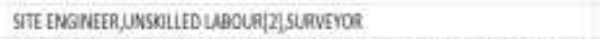 & 2103 in2.6a & \\
\hline 20 & ERCANATDN & 5 daps & Tue $0404-12$ & Mon 10-C4-17 & $\gamma_{30300000}$ & 16 & 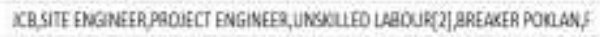 & $04-04 \$ 10-04$ & \\
\hline$n$ & $\begin{array}{l}\text { PAEPARATION OF PCC WORKS FOR } \\
\text { FOUNDATION }\end{array}$ & 14 depa & Tue $11 \cdot 04 \cdot 17$ & Fin $23-04-17$ & रु $3,000,000$ & & & $\Leftrightarrow$ & \\
\hline 22 & chessing of STMEA & $2 \mathrm{daps}$ & Tue $1184: 17$ & Wed $1204-17$ & ₹6,00000 & 20 & SOLED WAOUR|S|STIE ENGNEER & $1104 i 1204$ & \\
\hline 23 & Soiva & 1 idy & The $1304-17$ & The $11-04-17$ & 56,30002 & 32 & 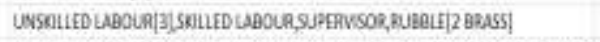 & 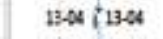 & \\
\hline 24 & SUITEGIMG FOR PCC & Sdans & Fri140417 & The $20.04 \cdot 17$ & $\xi_{14} \pi 0.00$ & 23 & 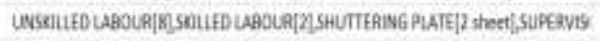 & 1004,2000 & \\
\hline 25 & DOUFING OF PXC & $4 d x$ & 대 21-04-1) & Wed $2604-17$ & $\$ 4,20000$ & 24 & 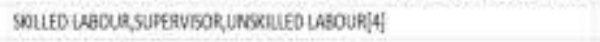 & 21.0472604 & \\
\hline is & DESHUTTESNS & 2 daps & Thu 27.0417 & Fir 28041$]$ & 8280000 & 25 & 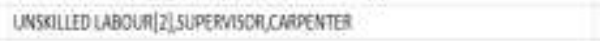 & $27.04 i 28.04$ & \\
\hline 27 & PREPARATION OF FOUNOATION & 12.5 daps & Mon 01-05-17 & Wed $17-05-17$ & है $410,450,00$ & & & 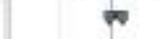 & \\
\hline
\end{tabular}

Fig -2: Screenshot 


\begin{tabular}{|c|c|c|c|c|c|c|c|c|c|c|}
\hline & 0 & Trate, & Thi Nate & $\begin{array}{l}\text { Athal } \\
\text { Anrmon }\end{array}$ & Wat. & Fint & howlont & Predar. & Asure Kras & 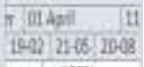 \\
\hline D. & $\forall$ & 8 & - paEfatanoN Of FOUKDation & 125 daps & YenOt-0s-1s & Wod 12-25-17 & के 422.450000 & & & w 1006 \\
\hline II. & 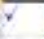 & "3. & LANOUT WHEKG & 1 tay & $\operatorname{Man} 0145: 17$ & Nonotos:1] & 83,20100 & 26 & 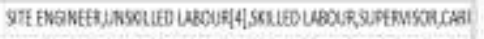 & 1006 \\
\hline s. & 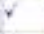 & a & LANNS OF RENKREEVEN & Ital & $\operatorname{Ta2} 92-\sqrt{5}-17$ & The $02 \cdot(5-27$ & \$83,5000 & 28 & 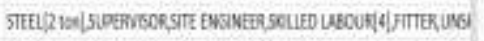 & 1000 \\
\hline 10. & $\checkmark$ & 5 & GUTTERKG FCG FOUNQATION & 15 ders & Wet $0145-17$ & The $0105-27$ & 83,52000 & 29 & 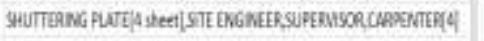 & isom \\
\hline 11. & 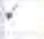 & 8 & MOSKG OF CONCEFIE & taty & Thasos-1] & Fick:-16.12) & F 133,4200 & 30 & 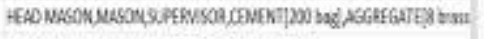 & fank \\
\hline 12. & $\checkmark$ & 8 & OESWITHANS & Ifal & fries 5 -1? & Nonasos-1) & $\delta \operatorname{tsnax}$ & \# & 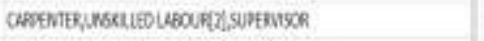 & i 1008 \\
\hline 13. & $\checkmark$ & 5 & Masevo ror couUn & Itay & Won $18-15-17$ & The $00-15-17$ & 5200000 & $\mathrm{n}$ & 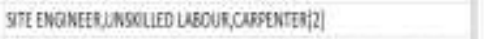 & $i \sin$ \\
\hline 14. & $r$ & 4 & $\begin{array}{l}\text { LHWG OFAEMFREMENT PCR } \\
\text { COLUN }\end{array}$ & taty & Toe $0705-17$ & Wed 10-05-1J & 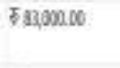 & 31 & 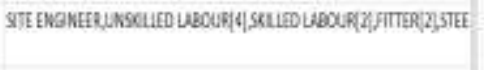 & $\mathrm{i} 1 \mathrm{~kW}$ \\
\hline 15. & $\checkmark$ & * & SUITHEG FCH COUWW & $2 \tan$ & Wed 1005:1? & Friles. & Estonto & 34 & 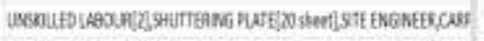 & $i 1000$ \\
\hline 16. & $\checkmark$ & 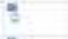 & NORKG OF COWCEETE & 2 an & $7122-17$ & $T=16-66-7)$ & 5rasinco: & 35 & 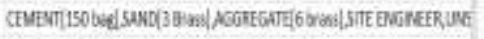 & $i 100 \%$ \\
\hline v. & $\checkmark$ & 5 & GESHUTEWWG & Itay & Fue $150-15-17$ & Wed 17-06-15 & 8200120 & 36 & 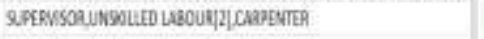 & inen \\
\hline 38 . & $\cdot$ & 4 & PUNTR woars & 21 ays & Wed $17 \cdot 65 \cdot 27$ & Thu $15-04-17$ & $5422,600.00$ & & & $\Rightarrow 1000$ \\
\hline 15. & $\checkmark$ & 8 & SETTESUPLEEL FOR FUKTH & Ifar & wed 1205.17 & The $18(6-2)$ & $\$ 3,3020$ & y & 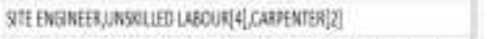 & toms \\
\hline 40. & $\checkmark$ & 5 & 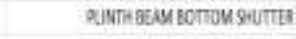 & 12 tap & Anv $5905-17$ & $\operatorname{Non} 22-05-15$ & 55,5000 & 39 & 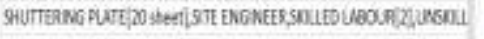 & fites \\
\hline 4. & $\cdot$ & 4 & LAMWGOE REARDOEMENT & 2 tap & Mat 22.45-17 & Wed 2*06-1J & 7155,2000 & 40 & 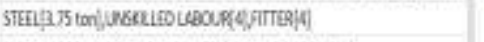 & $i \operatorname{tos}$ \\
\hline 4. & 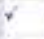 & 8 & LANNSOF SERCEINE & If & and 2435.17 & The $x(5,1)$ & 620010 & 41 & 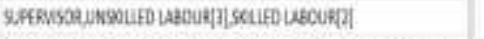 & (3006 \\
\hline 4. & $\checkmark$ & s & SOE SHUITERME FOR EEANS & $2 \tan$ & Dab-51] & $\operatorname{Non} 20(0-1)$ & DIL,300 & 42 & 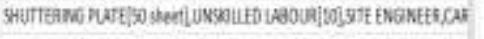 & 1000 \\
\hline 4. & $r$ & 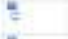 & MOANG DF CONGIETE & 2 lap & Mos $29-15 \cdot 17$ & Wed $3105-17$ & 5ु:57,50000 & 43 & 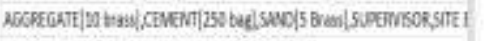 & i2006 \\
\hline 45. & $r$ & 8 & CESHITEUNE & $2 \tan$ & aed $3140-17$ & Friete-66-17 & 53,2020 & 4 & 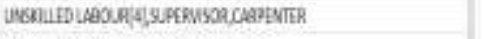 & (1000s \\
\hline 46. & $\checkmark$ & 7) & 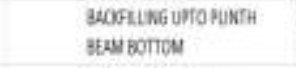 & thay & 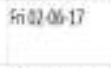 & $\operatorname{Non} 0 S \alpha-1 \gamma$ & 513,4000 & 45 & 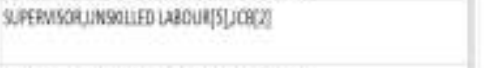 & zan \\
\hline a. & $\checkmark$ & s & coMPaction of sol & 2 lap & Won $05-05-17$ & Wedor-06-15 & 5.4,2000 & 46 & 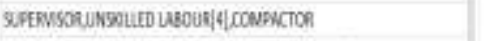 & $i 1006$ \\
\hline 48. & : & 4 & nusece soun: & 2 tap & Med 0T-Sh-17 & ficos 06.17 & F tag, sonco & 47 & 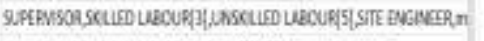 & {$[200 \%$} \\
\hline 4 5. & 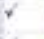 & 8 & LANWGOSM,FLM & Ifin & fries $0 \mathrm{r}-1$ ? & Nont20 $06-13$ & Finato & 48 & 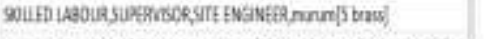 & $1100 \%$ \\
\hline 50 & $r$ & 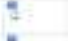 & LATINDOEPCC & 2 tan & Wos $12 \%-17$ & Wed 1400-1) & E31, 20010 & 49 & 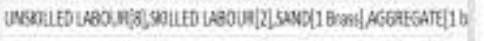 & íns \\
\hline 51. & $r$ & 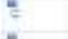 & LMNSOS DPC & Iten & Wet $14-25-17$ & Thu15-26-97 & 55,9000 & 50 & 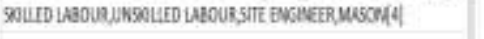 & 1000 \\
\hline g & & 8 & - SUPLSTRUKTUR! & 112 tan & The $15-(6-1)$ & Fnos-12-12 & 72528,03100 & & & \\
\hline \$1 & & 7 & - act wores & 112 àn & The $15-06-1)$ & finces 12,1$)$ & 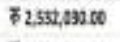 & & & \\
\hline S. & & 4 & - काषा & $112 \tan$. & Ten:15,65,17 & Bana-13. & 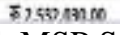 & & & \\
\hline
\end{tabular}

Fig -3: Contd. MSP Screenshot

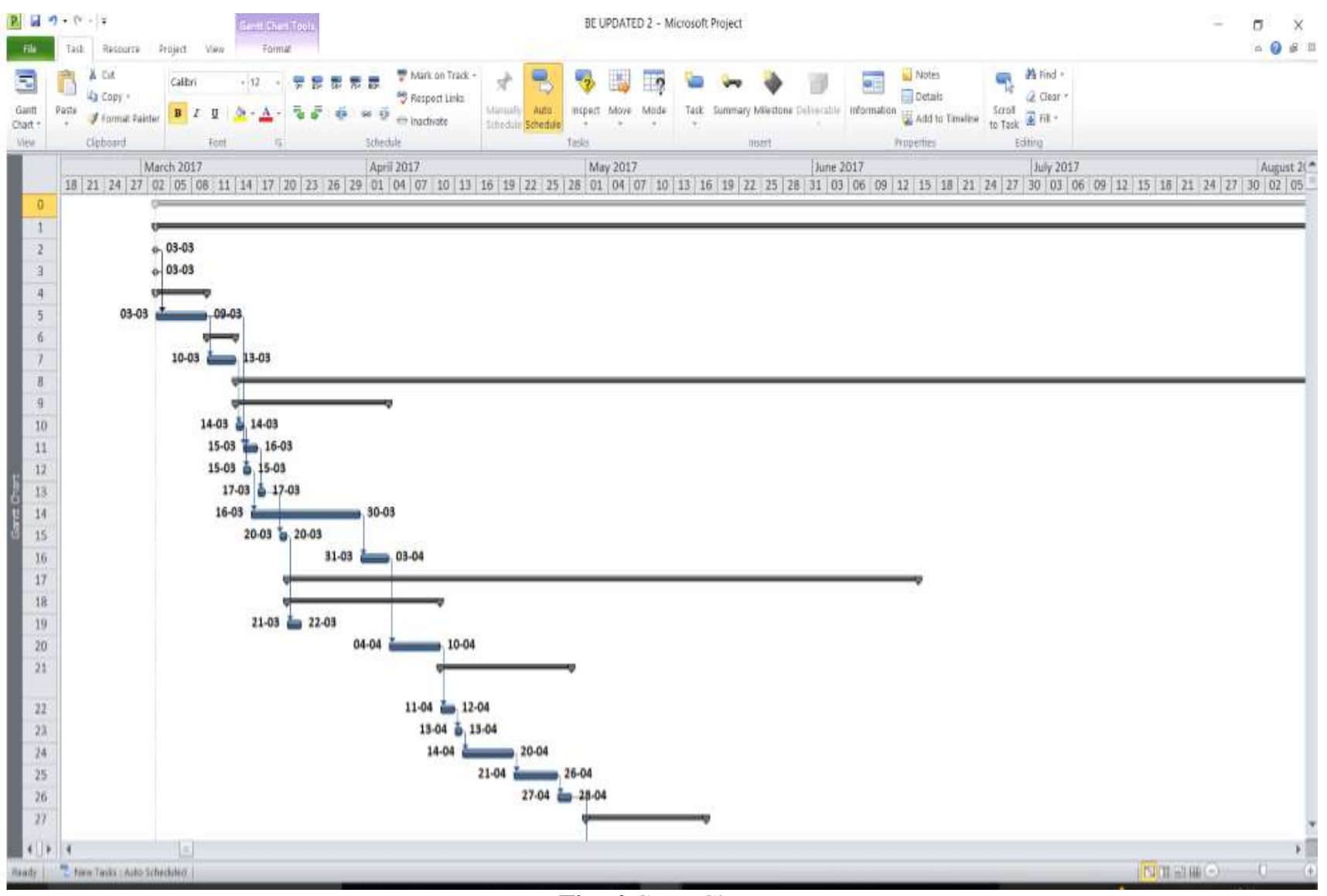

Fig -4:Gantt Chart 


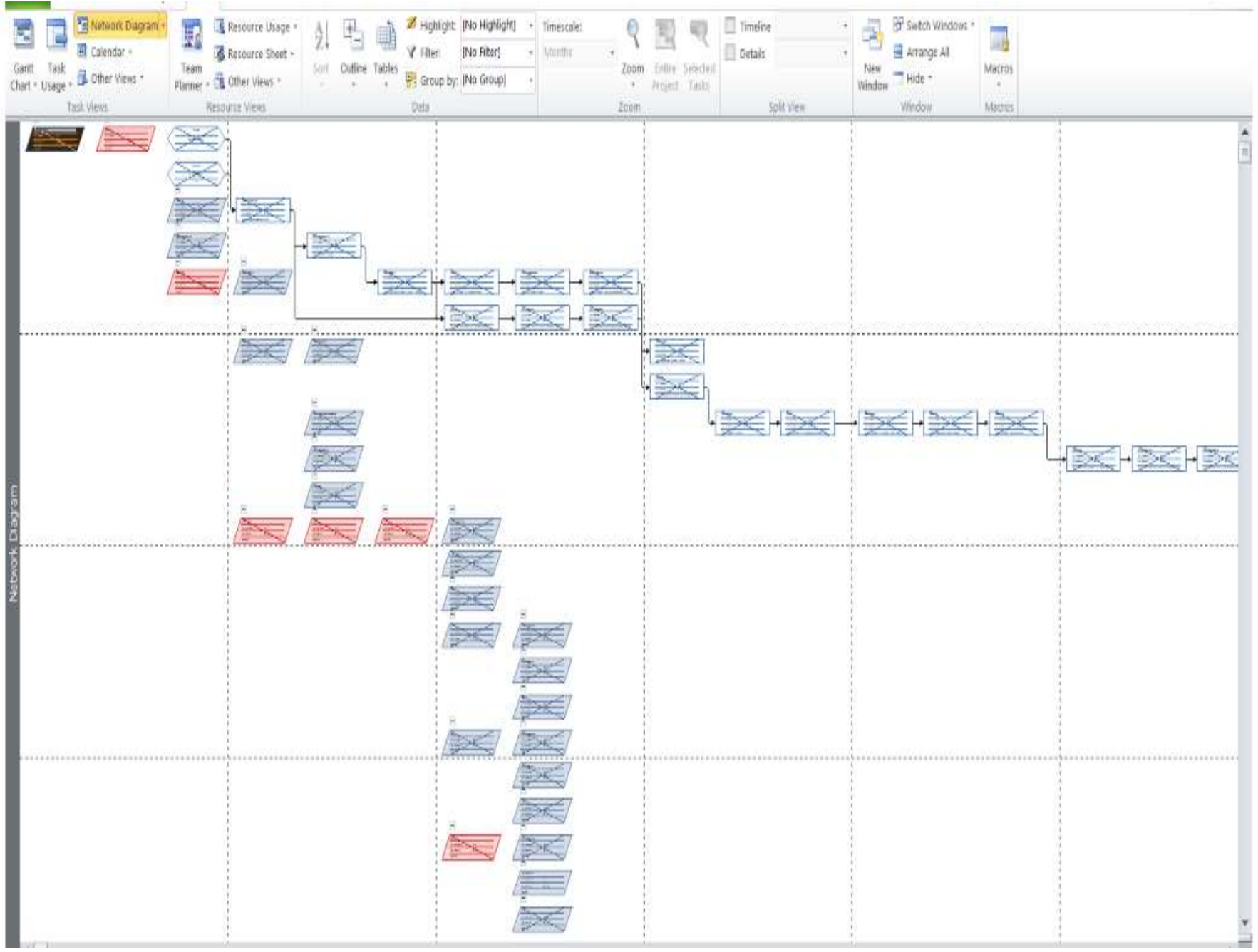

Fig -5: Network Diagram

\subsection{Calendar}

The Project calendar is basically used to establish the days and time for which the project will running. It also helps in establishing and assigning tasks to particular days. It also helps in assigning in resources to particular tasks for the respective days. So, by looking at the project calendar, one can clearly tell that on which days the organization or the construction company will be working on its project and on which day no work will be going on. MS Project 2010 has basically three base calendars. So for setting a base calendar for any project, either of the three base calendar decided by MSP 2010 can be chosen or own base calendar can also be created. A base calendar can be defined as a calendar template that can be applied to a set of resources, a set of tasks, or the project as a whole.

Following are the three base calendars that are defined by the MS Project 2010:

1. Standard base calendar: According to this base calendar, working days are from Monday to Friday and timing followed is 9 am to $6 \mathrm{pm}$. According to this calendar, lunch timing will be from 12 noon to $1 \mathrm{pm}$. This is the default base calendar used for the project, for task, and for resources.
2. Night shift base calendar: According to this calendar, working days will be from Monday to Friday and working timing will be from 11:00 pm to 8 pm with one hour off from 3 am to $4 \mathrm{am}$. It is basically used to work for the projects that will be carried on at night shifts.

3. 24 hours base calendar: According to this calendar, one has to work from midnight to midnight for seven days a week. This base calendar is usually practiced by manufacturing companies.

Any of the three above mentioned base calendars can be used as resource calendar or project calendar or task calendar or all the three calendars can be used at a time. 


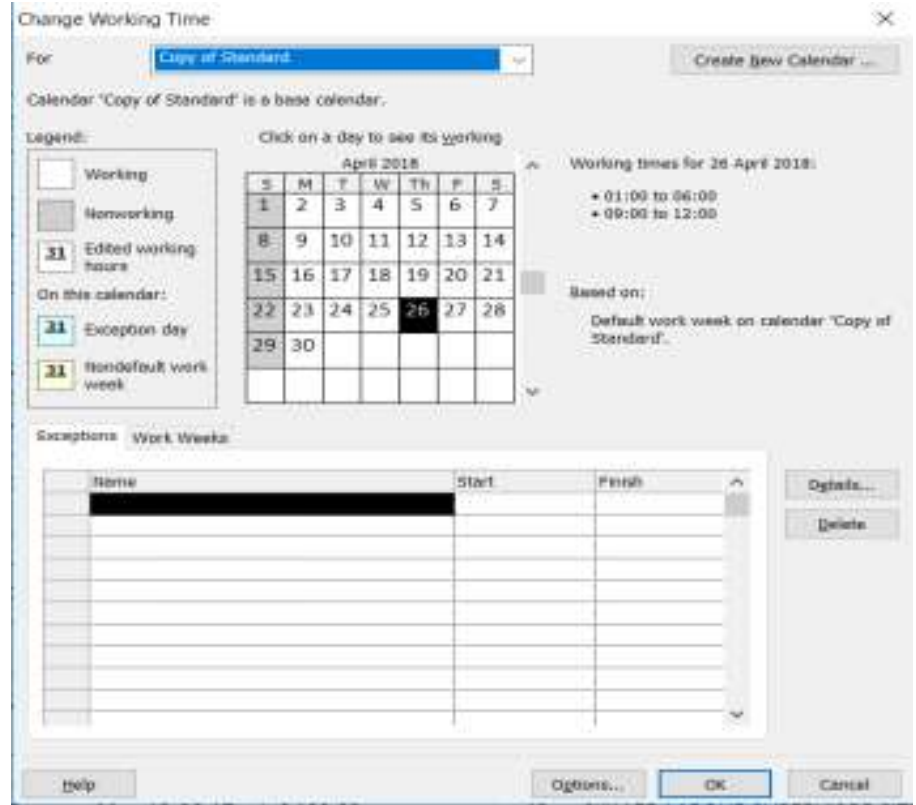

Fig -6: Creating Calendar

\subsection{Resources}

Resources are the materials or consumables or labors that are used to complete the tasks allocated to the project. These also define the expenditure or cost that will be incurred for the materials used in the project. Resources can help in following way:

- It helps in increasing the accuracy of a project. In case of 'Automatic Scheduling' selected, MSP 2010 will take into account the project calendar, task dependencies and duration and an accurate model of the project will be ready. After assigning resources to the project, MSP 2010 adds working time and availability of resources to the scheduled planned.

- It also helps in letting us to know, well ahead of time, the overloading of the resources at any stage of the project as well if there are any tasks that are under allocated. Later, at the time of work progress, information regarding task progress can be generated and the requirement of any new under allocation or over allocation can be identified.

- It can also help in tracking progress of the project according to resource work. It can also indicate how much time have been spent by a resource or how much time will be needed by it, this will be helpful in making any necessary adjustments so that the project can keep moving in the right direction. It will also be helpful in capturing actual progress data that can be used as historical information for the future projects.

- It helps in recording the cost, amount of materials consumed and also their use in the project. This will be beneficial for the well monitoring of the budget performance and also about the requirement of the record supplies well in advance.

- It is helpful in tracking some of the largest expenses in project such as labor cost, material cost, equipment cost, transportation cost etc.
- It will assure that proper resource allocation is followed and nothing is slipped through the cracks.

The following types of resources can be assigned to the tasks:

1. Work Resources: this includes equipment or people.

2. Material Resources: these include consumable materials.

3. Cost Resources: these include cost of the items or consumables incurred in performing a task.

\begin{tabular}{|c|c|c|c|c|c|c|}
\hline 2 & Fesocerlase & Tpes & Vaterit $\lfloor$ tes & hotas & Narlint soting & \\
\hline 1 & $\mathrm{xB}$ & Wor & & t & 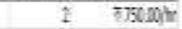 & \\
\hline 2 & UERUED UaSR & Wat & & U & 745000 itas & \\
\hline 3 & gUE 130.3 & With & & $s$ & $750000 / 6$ & \\
\hline 4 & Cusparis. & Wat & & c & Trabolar: & \\
\hline 5 & मान्द? & Wot & & f & 755000 las & \\
\hline 6 & BECTNOTAM & Wort & & E & $17100000,6 a$ & \\
\hline T & WESOC & Wot & & $M$ & 87100000 las & \\
\hline 8 & FELONLSSM & Wot & & H & 6 F.12000016s: & \\
\hline 9 & TrEerGaEB. & Writ & & 5 & 1 Fi, 0000 las & \\
\hline I & SIFESUSOR & Wat & & 5 & Tranoy, ar & \\
\hline II & 340 & Naterdi & brest & 5 & 7706018 & \\
\hline II & AGGSEALE & Natrod & bari & A & B150000 & \\
\hline II & caler & Narrid & bes & $c$ & 733008 & \\
\hline it & STER & Natrid & $\tan$ & 5 & racked & \\
\hline B & AQHITLUSE & Wat & & A & $175,00000,6 \mathrm{ar}$ & \\
\hline Is & LEDGN EIGAED, & Wot & & D & 1 r5,00000, bar & \\
\hline II & FOECTEVGEAR & Wat & & F & I. 7200000,60 & \\
\hline 2 & WATHNA & Wor & & u & $7300001 \mathrm{as}$ & \\
\hline$B$ & AHTECEVET & Netrit & & u & 712008 & \\
\hline$x$ & Ruver & Wit & & F & $2 \pi 50000 / 6$ & \\
\hline I1 & MITEW:PATE & Natrid & ther & 5 & 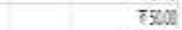 & \\
\hline II & DAL WGEPFE & Naterid & pertete & D & 730000 & \\
\hline 프 & WAB & Naterid & perters & y & 720008 & \\
\hline 34 & POQAN & Wok & & $p$ & $71100 \mathrm{phr}$ & \\
\hline 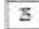 & BELERPOCA & Wot & & B & $71,30000 / \mathrm{r}$ & \\
\hline$x$ & detras & Wit & & $t$ & 1 Tssoogles & \\
\hline$\pi$ & SALIEC4 & Wort & & 5 & 1. 7 6asomear & \\
\hline 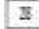 & Covproses & Wat & & c & $76 \times 0 \times 1$ & \\
\hline 3 & $\operatorname{non} x$ & Naterd & bat: & n & Foreco & \\
\hline 30 & P.SBLE & Natrit & Baㅐ & g & 3200008 & \\
\hline
\end{tabular}

Fig -7: Resource Sheet

\subsection{Critical Path and Critical tasks}

Determination of critical path is very important for the optimization of the project plan. It is well known that any kind of adjustments to the critical path can lead to the change of project's finish date resulting in changes in the resources, budget and scope of the project. So it becomes very important to determine the critical path as well as the critical tasks of every project using appropriate and proper methods. Here, in this project we have used CPM and PERT methods for the determination of critical path. 


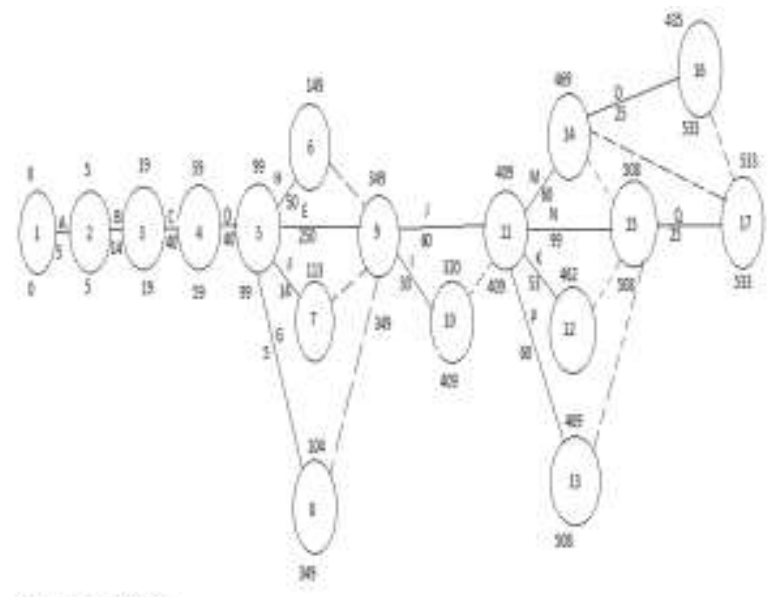

Table -6: PERT Chart

Fig -8: CPM Network

Table -6: CPM Chart

\begin{tabular}{|l|l|l|}
\hline & ACTIVITY & DURATION \\
\hline A & Document review & 5 \\
\hline B & Study of plan & 14 \\
\hline C & Site work & 40 \\
\hline D & Excavation work & 40 \\
\hline E & RCC frame work & 250 \\
\hline F & Underground water tank & 14 \\
\hline G & Septic tank & 5 \\
\hline H & Brick work & 50 \\
\hline I & Internal plastering & 30 \\
\hline J & External plastering & 60 \\
\hline K & Walls and floor tiles & 53 \\
\hline M & Electrical wiring & 60 \\
\hline N & Painting & 99 \\
\hline O & Fixing doors \& windows & 25 \\
\hline P & Plumbing & 60 \\
\hline Q & Finishing & 25 \\
\hline
\end{tabular}

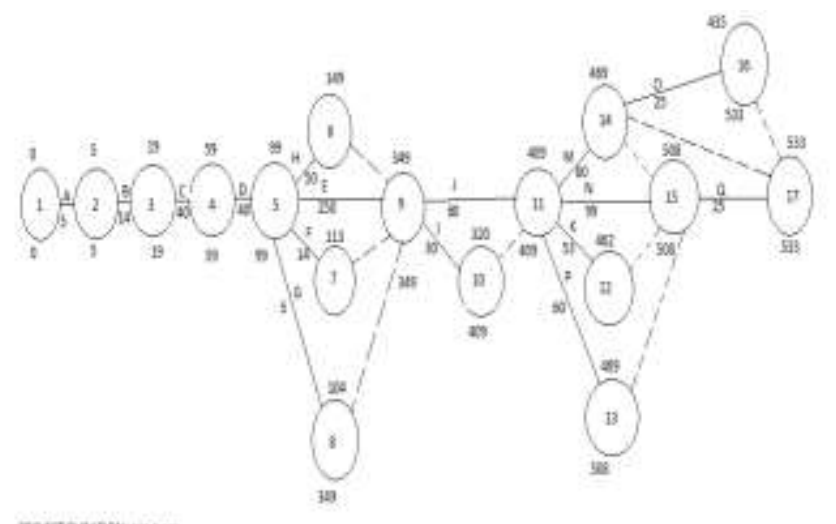

\begin{tabular}{|l|l|l|l|l|l|l|}
\hline Activity & To & Tp & Tm & Te & $\begin{array}{l}\text { s.d=(tp- } \\
\text { to)/6 }\end{array}$ & Variance \\
\hline A & 4 & 5 & 6 & 5 & 0.1667 & 0.0277 \\
\hline B & 13 & 14 & 15 & 14 & 0.1667 & 0.0277 \\
\hline C & 39 & 40 & 41 & 40 & 0.1667 & 0.0277 \\
\hline D & 39 & 40 & 41 & 40 & 0.1667 & 0.0277 \\
\hline E & 249 & 250 & 251 & 250 & 0.1667 & 0.0277 \\
\hline F & 13 & 14 & 15 & 14 & 0.1667 & \\
\hline G & 4 & 5 & 6 & 5 & 0.1667 & \\
\hline H & 49 & 50 & 52 & 50 & 0.1667 & 0.0277 \\
\hline I & 29 & 30 & 31 & 30 & 0.1667 & \\
\hline J & 59 & 60 & 61 & 60 & 0.1667 & \\
\hline K & 52 & 53 & 54 & 53 & 0.1667 & \\
\hline M & 59 & 60 & 61 & 60 & 0.1667 & 0.0277 \\
\hline N & 98 & 99 & 100 & 99 & 0.1667 & \\
\hline O & 24 & 25 & 26 & 25 & 0.1667 & \\
\hline P & 59 & 60 & 61 & 60 & 0.1667 & \\
\hline Q & 24 & 25 & 26 & 25 & 0.1667 & 0.0277 \\
\hline & & & & & & 0.2216 \\
\hline
\end{tabular}

$X=\sqrt{ }$ variance $=0.471$

$>$ Expected days 533 days

$\mathrm{Z}=($ te-tp $) / \mathrm{x}=(533-535) / 0.471=-4.252$

Probability $=0.3 \%$

$>$ Probability of completion of project in 692 days

$\mathrm{Z}=($ te-tp $) / \mathrm{x}=(537-535) / 0.47=4.252$

Probability $=98.3 \%$

$>$ If probability is $90 \%$, what is the project duration $\mathrm{Z}=($ te-tp $) / \mathrm{x}, 1.5=($ te-tp $) / 0.471=530$ days

\section{CONCLUSION}

In the project of planning and scheduling we had selected the site located near Karjat, Mumbai, India. The building is under construction. The estimated time required to complete this building as per their planning is 20 months. We intended to make the study of this project as the case study of our project work. Hence attempt is made for planning and scheduling as a case study of our project.

We had used MS software for planning and scheduling of material as well as labour, which gives us brief information about the activities in construction project, showing their start and finish time, and material and labour requirement as well as project duration.

We have done CPM and PERT analysis for Planning. The result obtained by our planning shows that the estimated time of completion of this project is 18 months, highlighting the critical activities and critical path duration required for completion for some activities are more than software result

Fig -9: PERT Network 
which indicate that some activities are delayed by 4 months. We have done resource allocation for those labour, material and activities which are delaying the project, then we calculated the time required for completing the project using software. Then the comparison between the result obtained by the software and on actual site was done.

Hence, it was concluded that time required for completing the project as per our planning and scheduling is 4 months prior to their planning and scheduling. Hence our method of planning and scheduling is more scientific.

\section{REFERENCES}

[1] Rhuta Joshi and V.Z. Patil (2013) "Resource scheduling of construction project." International journal of science and research, Volume 4, Issue 5, PP.563-568.

[2] Wallace Agyei (2015) "Project Planning and Scheduling using PERT and CPM techniques with linear programming." International journal of scientific and technology research, Volume 4, Issue 8, PP.222227.

[3] P. M. Wale and N. D. Jain (2015) "Planning and scheduling of Project using Microsoft Project." IOSR journal of mechanical and civil engineering (IOSRJMCE), Volume 12, Issue 3, PP. 57-63.

[4] Monish Kumar and Maheshwar S. Margoundra (2017) " Construction project scheduling of M. K. Apartments using MS Project 2013, International research journal of Engineering and Technology (IRJET), Volume 4, Issue 7.

[5] Nikhil R. Mahajan, M. V. Bhogone (2017) "Resource scheduling in construction project using MSP". International journal of innovative research in science, Engineering and Technology volume 6, Issue 9. 